\title{
National Burnout Trends Among Physicians Working in the Department of Veterans Affairs
}

\author{
Seppo T. Rinne, MD PhD 1,2, David C. Mohr, PhD ${ }^{3,4}$, Lakshman Swamy, MD MBA2, \\ Amanda C. Blok, MSN PhD ${ }^{7,5}$, Edwin S. Wong, PhD ${ }^{6,7}$, and Martin P. Charns, MBA, DBA 3.4
}

${ }^{1}$ Center for Healthcare Organization \& Implementation Research, VA, Bedford, MA, USA; ${ }^{2}$ The Pulmonary Center, Boston University School of Medicine, Boston, MA, USA; ${ }^{3}$ Center for Healthcare Organization \& Implementation Research, VA, Boston, MA, USA; ${ }^{4}$ Department of Health Law, Policy, \& Management, Boston University School of Public Health, Boston, MA, USA; ${ }^{5}$ College of Nursing and Health Sciences, University of Massachusetts, Boston, MA, USA; ${ }^{6}$ Seattle-Denver Center of Innovation for Veteran-Centered \& Value-Driven Care, VA Puget Sound Health Care System, Seattle, WA, USA; ${ }^{7}$ Department of Health Services, University of Washington, Seattle, WA, USA.

BACKGROUND: Heterogeneity of existing physician burnout studies impairs analyses of longitudinal trends, geographic distribution, and organizational factors impacting physician burnout. The Department of Veterans Affairs (VA) is one of the largest integrated healthcare systems in the USA, offering a unique opportunity to study burnout across VA sites and time.

OBJECTIVE: To characterize longitudinal burnout trends of VA physicians and assess organizational characteristics and geographic distribution associated with physician burnout.

DESIGN: Longitudinal study of the VA All Employee Survey during 2013-2017.

PARTICIPANTS: Self-identified physicians practicing in one of nine clinical service areas at 140 VA sites nationwide.

MAIN MEASURES: We identified burnout using a validated definition adapted from the Maslach Burnout Inventory and characterized burnout trends for physicians in different clinical service areas. We used clustering analysis to categorize sites based on their burnout rates over time, and compared organizational characteristics and geographic distribution of high, medium, and low burnout categories.

KEY RESULTS: We identified 40,382 physician responses from $140 \mathrm{VA}$ sites. Mean burnout rates across all physicians ranged from $34.3 \%$ in 2013 to a high of $39.0 \%$ in 2014. Primary care physicians had the highest burnout. High burnout sites were more likely to be rural and non-teaching, have lower complexity (i.e., offer fewer advanced clinical services), and have fewer unique patients per site.

CONCLUSIONS: VA physician burnout was lower than previously described in many non-VA studies and was relatively stable over time. These findings may be due to unique characteristics of the VA practice environment. Nonetheless, with over a third of VA physicians reporting burnout, organizational interventions are needed. Primary care physicians and those practicing at small, rural

Electronic supplementary material The online version of this article (https://doi.org/10.1007/s11606-019-05582-7) contains supplementary material, which is available to authorized users.

Received May 16, 2019

Accepted November 25, 2019

Published online February 24, 2020 sites have higher rates of burnout and may warrant more focused attention. Our results can guide targeted interventions to promote VA physician well-being and inform efforts to address burnout in diverse clinical settings.

KEY WORDS: physician burnout; Veterans Affairs; longitudinal trends.

J Gen Intern Med 35(5):1382-8

DOI: $10.1007 / \mathrm{s} 11606-019-05582-7$

(c) Society of General Internal Medicine (This is a U.S. government work and not under copyright protection in the U.S.; foreign copyright protection may apply) 2020

\section{INTRODUCTION}

A growing body of literature implicates physician burnout as a threat to patient care, with previous studies demonstrating associations between burnout and worse clinical outcomes, lower patient satisfaction, and major medical errors. ${ }^{1-3}$ While definitions of burnout vary, it is commonly described as a work-related syndrome involving feelings of emotional exhaustion, depersonalization, and lack of personal accomplishment. ${ }^{4}$ Physicians suffering from burnout have higher rates of depression, substance abuse, and suicidal ideation, ${ }^{5-7}$ and they are more likely to retire early or leave medicine..$^{8,9}$

Despite the risk that burnout poses to physicians and patients, existing literature has been limited, with few longitudinal studies on burnout trends. In a series of investigations, Shanafelt et al. compared national US burnout rates across multiple physician specialties in 2011, 2014, and 2017 and found overall burnout rates of $45.5 \%, 54.4 \%$, and $43.9 \%$ respectively. ${ }^{10-12}$ These trends have not been examined in other studies, nor have they been contrasted across healthcare environments. A systematic review of burnout studies found excessive heterogeneity of burnout definitions, assessment methods for burnout, and study quality that impaired pooling burnout measures across studies to evaluate longitudinal trends, geographic distributions, and organizational characteristics associated with burnout. ${ }^{13}$

The Department of Veterans Affairs (VA) is one of the largest integrated healthcare systems in the USA with more than 11,000 physicians nationwide. ${ }^{14}$ The VA annually conducts the All Employee Survey (AES) to assess perceptions of 
practice environment and employee satisfaction. ${ }^{15}$ Since 2013, the AES has incorporated questions from the Maslach Burnout Inventory (MBI), including feelings of emotional exhaustion, depersonalization, and personal accomplishment. ${ }^{4}$ Given the standardized methods among a national sample of physicians over a longitudinal period, the common VA work setting, and the ability to drill down to respondents' site and service area, the AES data provides a unique opportunity to study trends in burnout and organizational characteristics associated with changes in burnout over time.

Our objective was to characterize longitudinal burnout trends of VA physicians, categorize VA sites based on burnout trends, and assess organizational characteristics, including geographic distribution of physician burnout. Results of this research could provide insight into target populations and interventions to improve physician well-being.

\section{METHODS}

\section{Study Design}

We conducted a nationwide longitudinal panel study of AES responses from 140 VA medical facilities during the period of 2013-2017. In panel study designs, repeated measures are collected from a population of interest at specific time intervals, as is done through annual surveys. ${ }^{16}$ The institutional review board at The VA Boston Healthcare System approved the study.

\section{Survey Instrument}

The AES is an anonymous annual census survey that assesses employee demographics, job satisfaction, attitudes, and perspectives of practice environment. Each VA medical center has an AES coordinator who is responsible for identifying VA employees and designating them to unique workgroups that represent localized units of interest for reporting purposes. All active VA employees are invited to respond to the AES via email link to a secure online platform. The total number of eligible and invited Veterans Health Administration (VHA) employees ranged from 284,634 (2013) to 330,732 (2017) and the national response rate ranged from 56 to $60 \%$.

\section{Study Population}

The AES asks respondents to select their occupation from a comprehensive list of VA positions, such as physicians, dentists, physician assistants, nurse practitioners, medical support assistants, and research staff. Respondents are then asked to select their primary service from a list of 21 clinical and nonclinical options. We limited our sample to respondents who indicated they were a physician whose primary service was in one of nine core areas: Primary Care, Medical Specialty, Emergency Medicine, Inpatient Medicine, Surgery, Anesthesiology or Surgical Specialty Care, Mental Health, Imaging, Laboratory and Pathology, and Rehabilitation Services. We selected these service areas because of their clinical relevance and robust sample sizes across years. These categories roughly parallel American Council for Graduate Medical Education (ACGME) specialties.

\section{Burnout Definition}

We identified physicians with burnout based on responses to single-item MBI statements about emotional exhaustion ("I feel burned out from my work") and/or depersonalization ("I worry that this job is hardening me emotionally"). ${ }^{4}$ Multiple-choice responses to these questions were listed on a frequency-based scale, including "never," "a few times a year or less," "once a month or less," "a few times a month," "once a week," "a few times a week," and "every day." Physicians responding that either of these statements were true once a week or more frequently were designated as having burnout, while those who indicated that both of these statements were true a few times a month or less frequently were designated as not having burnout. This commonly used approach has been validated against another standard definition that relies on dichotomized emotional exhaustion and/ or depersonalization domains from the full MBI. ${ }^{17}$ The primary outcome variable, site-level burnout, was constructed as the unadjusted proportion of physicians who reported burnout in a given year. Site-level burnout was measured for each service and year between 2013 and 2017.

\section{Organizational Characteristics}

To examine factors associated with site-level physician burnout rates, we identified organizational characteristics of each VA medical center using administrative data sources. We included site complexity based on available clinical services, such as intensive care, trauma, and advanced surgical services (level 1 sites are the most complex, and level 3 the least complex). ${ }^{18}$ We identified the average annual number of unique patients per site over the study period. We also identified location (city and state) for geographic mapping of burnout rates, categorized geography based on US Census region, and used the Rural-Urban Commuting Area (RUCA) system to define rurality (accounting for population density and proximity to urban centers). ${ }^{19}$ Finally, we identified teaching hospitals using the Association of American Medical Colleges (AAMC) Council of Teaching Hospitals. ${ }^{20}$

\section{Statistical Analysis}

We conducted descriptive analyses of national trends in VA physician burnout rates and presented these trends for each service area of interest. We conducted separate site-level analyses, for which we applied the clustering algorithm (Ward's minimum variance) to identify groups of similar sites based on trajectories of yearly burnout rates across all clinical service areas. ${ }^{21}$ We restricted the clustering analysis to facilities with at least 10 respondents per year to ensure stability in burnout estimates. We explored the variance explained by the different number of clusters $(k=2,3,4$, and 5$)$ and selected the number 
of clusters that best represented variation in the data. Using the resulting three-factor cluster pattern accounting for $85 \%$ of variance, we then computed chi-square tests to assess differences in organizational factors.

In secondary analyses, we conducted one-way ANOVA tests to examine differences in burnout by year for each service. We also used chi-square analysis and phi coefficients to examine individual and organizational characteristics associated with burnout across all years. This approach allows an analysis of characteristics associated with burnout among survey responses but does not account for the possibility that responses are not necessarily from unique individuals (i.e., the same physician may respond to AES multiple years in a row). Statistical analyses were performed using SAS Statistical Software (version 9.2) and geographic mapping was performed using R (version 3.5.3). ${ }^{22,} 23$

\section{RESULTS}

We identified 40,382 physician responses from 140 VA medical centers (an average of 8076 responses per year). Physician characteristics for each year are presented in Appendix 1. Mean physician burnout among all respondents was $34.3 \%$ $(\mathrm{SD}=47.5 \%)$ in $2013,39.0 \%(\mathrm{SD}=48.8 \%)$ in $2014,37.1 \%$ $(\mathrm{SD}=48.3)$ in $2015,36.8 \%(\mathrm{SD}=48.2 \%)$ in 2016 , and $37.3 \%$ $(\mathrm{SD}=48.4 \%)$ in 2017 . The mean year-to-year changes in sitelevel burnout was $8.4 \%$ ( $\mathrm{SD}=7.4 \%$ ). Primary care physicians had the highest burnout, followed by mental health and emergency medicine physicians (Fig. 1). Each service had a distinct burnout trend pattern over the study period. The only service

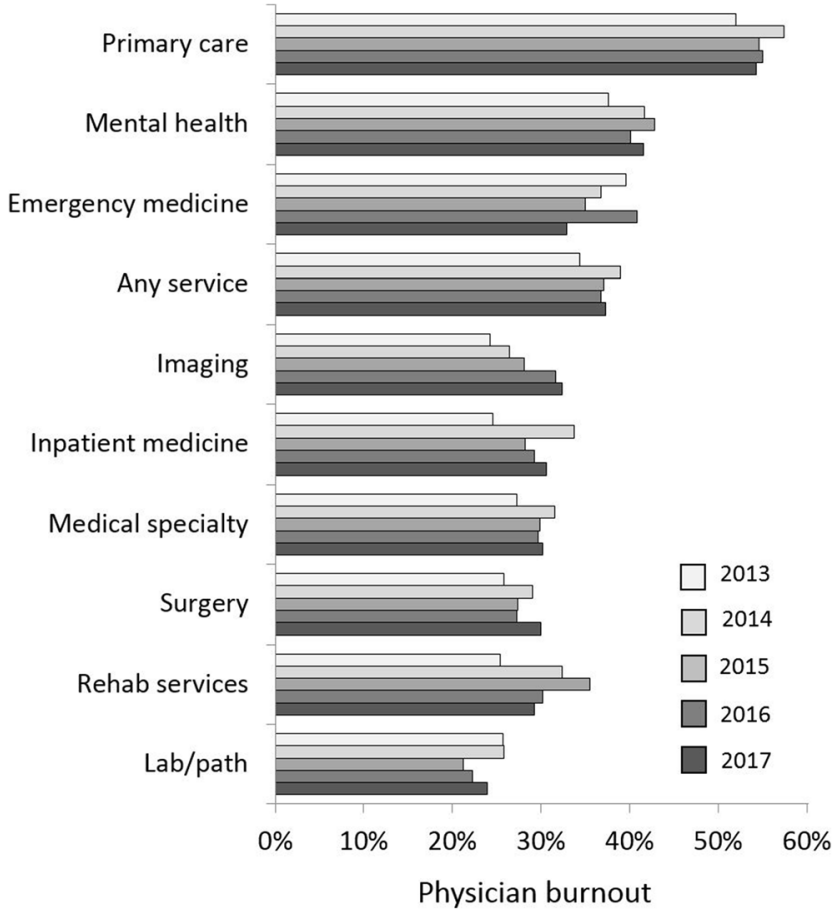

Figure 1 Trends in VA physician burnout by service area for the period of 2013 through 2017. to demonstrate consistently rising burnout rates over time was imaging, which had a burnout rate of $24.3 \%$ ( $\mathrm{SD}=42.9 \%$ ) in 2013 and rose to $32.4 \%(\mathrm{SD}=46.9 \%$ ) in 2017. Mean burnout and number of AES responses for each service area by year are reported in Appendix 2. Results show the largest differences among inpatient medicine, imaging, and primary care.

For clustering analysis, we examined 119 VA facilities with at least 10 responses in all years, representing 39,819 physician responses over the study period. Based on site-level burnout over time, we assigned sites to one of three clusters, which we labeled "low" $(n=56)$, "medium" $(n=40)$, and "high" $(n=22)$ (Table 1). We observed wide variation in site-level burnout both within and across clusters (Fig. 2).

Site-level characteristics differed significantly across burnout clusters. When compared with consistently low burnout sites, high burnout sites were more likely to be rural, nonteaching, have lower level of complexity (i.e., offering fewer advanced clinical services), and have fewer unique patients per site (Table 2). High and medium burnout sites also tended to be located in the South (Fig. 3) though Census region was not significant in chi-square analysis.

In secondary analysis, we identified multiple individual and organizational characteristics associated with burnout among survey respondents (Appendix 3). Notably, VA tenure was strongly associated with burnout, with the highest burnout among providers with 10-15 years of VA experience (44.5\%) and lower among physicians with $<6$ months of experience (18.4\%).

While primary care was the service area with the most AES responses, they only represented $22.7 \%$ of all responses. In subgroup analysis, the minority of rural-site responses were from PCPs (30.9\% of rural responses). Burnout rate for rural PCPs was $58.9 \%$ compared with $54.1 \%$ of urban PCPs $\left(\chi^{2}(1\right.$, $8933), p<.001, \varphi=.03)$. Similarly, the minority of nonteaching site responses were from PCPs $(29.9 \%$ of nonteaching responses). Burnout rates for PCPs in non-teaching facilities were $57.9 \%$ compared with $52.8 \%$ for primary care physicians in teaching facilities $\left(\chi^{2}(1,8933)=21.58, \varphi=.05\right)$.

\section{DISCUSSION}

This research represents the largest national study on physician burnout to date. Longitudinal data on burnout is critical to

Table 1 Mean Annual Burnout Rates Across Burnout Clusters

\begin{tabular}{llllll}
\hline \hline $\begin{array}{l}\text { Burnout } \\
\text { cluster }\end{array}$ & \multicolumn{4}{l}{ Burnout rate by year } \\
\cline { 2 - 6 } & $\begin{array}{l}\mathbf{2 0 1 3} \\
\text { (mean } \\
\text { (SD)) }\end{array}$ & $\begin{array}{l}\mathbf{2 0 1 4} \\
\text { (mean } \\
\text { (SD)) }\end{array}$ & $\begin{array}{l}\mathbf{2 0 1 5} \\
\text { (mean } \\
\text { (SD)) }\end{array}$ & $\begin{array}{l}\mathbf{2 0 1 6} \\
\text { (mean } \\
\text { (SD)) }\end{array}$ & $\begin{array}{l}\mathbf{2 0 1 7} \\
\text { (mean } \\
\text { (SD)) }\end{array}$ \\
\hline High & 46.5 & 57.7 & 56.4 & 46.6 & 46.0 \\
$(n=22)$ & $(9.3)$ & $(10.0)$ & $(12.1)$ & $(9.6)$ & $(9.5)$ \\
Medium & 37.8 & 42.2 & 39.2 & 44.6 & 43.2 \\
$(n=41)$ & $(6.8)$ & $(6.0)$ & $(7.4)$ & $(6.9)$ & $(10.7)$ \\
Low & 30.9 & 33.5 & 32.9 & 30.4 & 32.9 \\
$(n=56)$ & $(7.7)$ & $(6.8)$ & $(6.6)$ & $(6.7)$ & $(5.5)$ \\
\hline
\end{tabular}




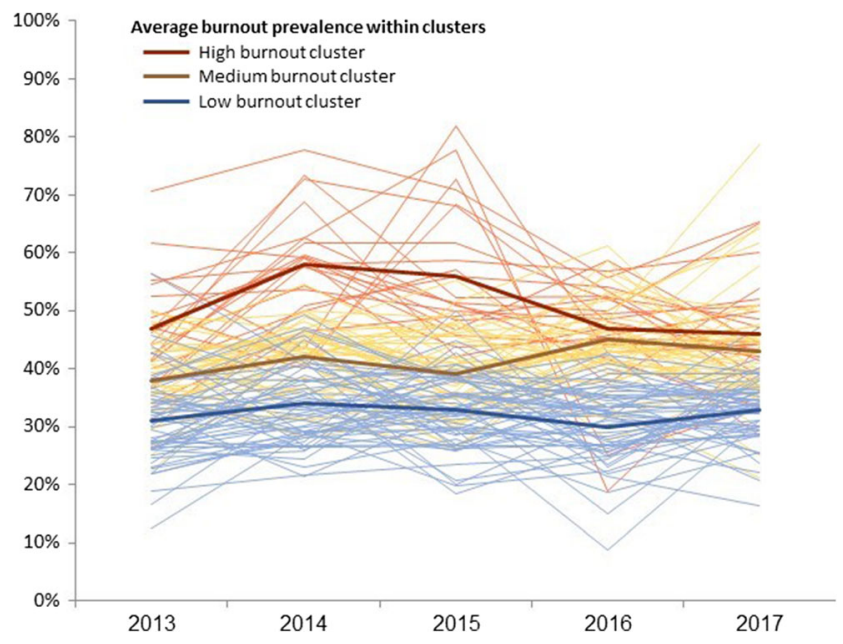

Figure 2 VA sites clustered by physician burnout.

monitor the health of physician workforce that is already experiencing shortages in many specialties. ${ }^{24}$ Staffing scarcities are particularly concerning in the VA, where recruiting and retaining physicians can be challenging. ${ }^{14,25}$ Our research on VA physician burnout can offer a comparison to the private sector experience and may indicate differences that inform interventions to reduce burnout. We characterized trends in VA physician burnout across nine clinical services, which produced key findings that differentiate our results from previous research, including (1) VA physician burnout is lower than previously described in many non-VA studies; (2) nationally, VA physician burnout rates were relatively stable over time; (3) VA primary care physicians experienced the highest burnout of any service area examined; and (4) site-level characteristics were associated with higher physician burnout, including small, rural sites, with lower complexity.
In the national studies by Shanafelt et al., physician burnout ranged from a high of $54.4 \%$ in 2014 to a low of $43.9 \%$ in $2017 .{ }^{10}$ The prevalence of burnout in our study was lower (ranging from 34\% in 2013 to $39 \%$ in 2014), which could reflect a true difference or may be the related to differences in measurement. The burnout prevalence that we identified is still concerning and warrants closer evaluation and management through organizational interventions.

The relatively lower prevalence of VA physician burnout may relate to differences in clinical responsibilities, workload, and workplace climate. For example, VA positions are often cited as having greater work-life balance relative to comparable jobs in the private sector. ${ }^{26}$ For primary care physicians, VA patient panels tend to be smaller than in the private sector. $^{27}$ Furthermore, VA represents a unique practice environment in which physicians do not experience the same financial and regulatory pressures that are present in other healthcare settings. ${ }^{28,29}$ VA physicians may also be engaged and motivated by the VA's mission and the Veteran population, leading to greater job satisfaction and lower rates of burnout.

We found that the nationwide prevalence of VA physician burnout was relatively stable over time. Very few studies have examined longitudinal trends in physician burnout, and most of this information comes from the nationwide studies by Shanafelt et al., discussed previously. ${ }^{10-12}$ In these studies, there was an initial concern for rapidly rising burnout rates (from $45.5 \%$ in 2011 to $54.4 \%$ in 2014), though the most recent study found the lowest burnout rate at $43.9 \% .^{10}$ The website Medscape tracks self-reported physician burnout through a large convenience sample of online survey responses and found physician burnout increased from $40 \%$ in 2013 to $51 \%$ in 2017 , but was $44 \%$ in the most recent 2019 report. ${ }^{30}$ In our analysis, we did not observe large changes in

Table 2 Site Characteristics of Burnout Clusters

\begin{tabular}{|c|c|c|c|c|}
\hline \multirow[t]{2}{*}{ Characteristic } & \multicolumn{3}{|c|}{ Burnout cluster } & \multirow[t]{2}{*}{$p$ value } \\
\hline & Low $(N=56)$ & Medium $(N=41)$ & High $(N=22)$ & \\
\hline & No. $(\%)$ & No. $(\%)$ & No. $(\%)$ & \\
\hline Setting & & & & $<0.01$ \\
\hline Rural & $2(4 \%)$ & $10(24 \%)$ & $7(32 \%)$ & \\
\hline Urban & $54(96 \%)$ & $31(76 \%)$ & $15(68 \%)$ & \\
\hline Teaching & & & & $<0.01$ \\
\hline Yes & $45(80 \%)$ & $19(46 \%)$ & $4(18 \%)$ & \\
\hline No & $11(20 \%)$ & $22(54 \%)$ & $18(82 \%)$ & \\
\hline Region & & & & 0.43 \\
\hline Midwest & $16(29 \%)$ & $9(22 \%)$ & $5(23 \%)$ & \\
\hline Northeast & $14(25 \%)$ & $5(12 \%)$ & $2(9 \%)$ & \\
\hline South & $18(32 \%)$ & $17(41 \%)$ & $10(45 \%)$ & \\
\hline West & $8(14 \%)$ & $10(24 \%)$ & $5(23 \%)$ & \\
\hline Complexity & & & & $<0.01$ \\
\hline Level 1 (most complex) & $46(82 \%)$ & $28(68 \%)$ & $9(41 \%)$ & \\
\hline Level 2 & $7(13 \%)$ & $9(22 \%)$ & $5(23 \%)$ & \\
\hline Level 3 (least complex) & $3(5 \%)$ & $4(10 \%)$ & $8(36 \%)$ & \\
\hline No. of patients/site & & & & 0.07 \\
\hline Q1一few & $4(7 \%)$ & $5(12 \%)$ & $7(32 \%)$ & \\
\hline Q2 & $12(21 \%)$ & $14(34 \%)$ & $6(27 \%)$ & \\
\hline Q3 & $19(34 \%)$ & $10(24 \%)$ & $6(27 \%)$ & \\
\hline Q4-most & $21(38 \%)$ & $12(29 \%)$ & $3(14 \%)$ & \\
\hline
\end{tabular}




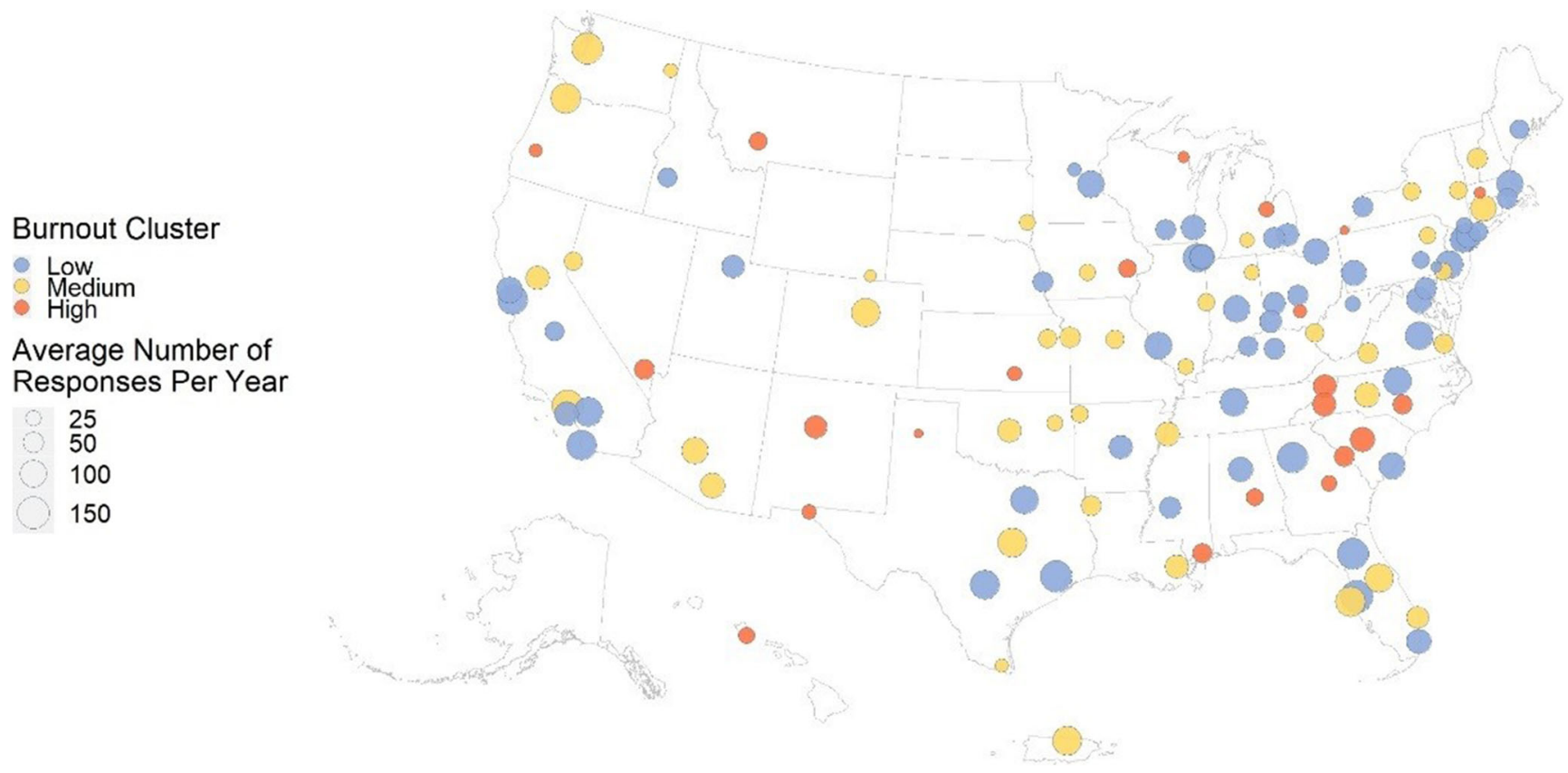

Figure 3 Geographic distribution of burnout across VA sites nationwide.

burnout during the 2013 to 2017 period. The modest increase in physician burnout from $34.3 \%$ in 2013 to $39.0 \%$ in 2014 was not sustained, and burnout rates returned to $37 \%$ for the remaining 2015-2017 study years. At the time that VA conducted the 2014 AES, national media coverage focused on challenges with access to VA care. ${ }^{31}$ Negative media coverage may impact physician morale and result in higher burnout. ${ }^{32}$ The otherwise stable burnout rates in VA suggest that changes in work-related stressors associated with varying burnout rates among non-VA physician do not appear to be present to the same degree or affect VA physicians in the same way. ${ }^{10}$

Primary care physicians in our study had the highest burnout of any service area followed by mental health and emergency medicine. Veterans are a patient population with disproportionate multimorbidity and complex psychosocial needs. ${ }^{33}$ To more effectively address the needs of these and other medically complex patients, VA restructured the delivery of primary care, adopting the Patient-Centered Medical Home model across all sites nationwide. ${ }^{34}$ Mental health providers have been integrated into Patient-Aligned Care Teams (PACTs) to provide comprehensive care for Veterans and rapid access to mental health services. This system redesign has improved quality of primary care and mental health services. However, previous research indicates that primary care providers exhibited greater turnover, burnout, emotional exhaustion, and challenges with insufficient staffing. ${ }^{35-38}$

Our data-driven analyses identified three distinct groups of facilities characterized by burnout levels over time. Several site-level characteristics distinguished sites with high burnout, including rural location, non-teaching status, lower site complexity, and fewer unique patients per site. Even among nonPCP responses, rural and non-teaching status were associated with a higher risk of burnout, suggesting that service area was not the only cause of higher burnout in these settings. These findings are consistent with previous research that found lower job satisfaction among physicians practicing at rural and small hospitals. ${ }^{39,}{ }^{40}$ Physicians at these sites may have more responsibilities for diverse aspects of patient care with less available support for complex patient needs. ${ }^{41,42}$

We found that sites with lower complexity and fewer unique patients had higher burnout, though we do not have information on staffing or individual physician responsibilities. It is possible that physicians practicing at these sites are relatively understaffed and have fewer resources to manage complex patient needs. These sites also tend to have more PCP responses, a group that we found to have higher burnout rates. Prior research has shown that higher patient load and patient severity of illness are associated with physician burnout, ${ }^{43}$ though we were not able to examine these characteristics on a physician level to determine whether they are risk factors for burnout in our cohort. Small and rural sites also tend to offer fewer opportunities for professional development and nonclinical pursuits, such as teaching and research, which may further contribute to burnout. ${ }^{44-46}$ Physician burnout in small, rural sites is particularly concerning, given the challenges recruiting and retaining physicians in these areas. ${ }^{47}$

The geographic location of VA sites based on US census region was not significantly associated with burnout cluster type, though there was an appearance of non-random distribution, with a tendency for low burnout sites to graphically be in the Northeast and high burnout sites in the South. This distribution may relate to differences in patient populations and healthcare factors. Patients located in the South tend to have more socioeconomic, behavioral, and metabolic risk factors that may complicate effective healthcare delivery and strain physician efforts. ${ }^{48}$ Differences in local leadership and 
organizational culture may also contribute to differences in burnout.

In our secondary analyses, we found highest burnout rates among physicians who have 10-15 years of VA tenure. This finding is consistent with other literature that demonstrates high burnout among mid-career physicians, who tend to work longer hours and have lower satisfaction with work-life balance. ${ }^{49},{ }^{50}$ Interventions to promote VA clinician well-being ought to consider career stages, particularly for mid-career physicians.

Our findings identified medical specialties and VA sites with high burnout rates, which could represent target populations for interventions to improve VA physician well-being. Existing research has established the importance of organizational interventions to reduce physician burnout. ${ }^{51} \mathrm{VA}$ administrators ought to ensure these interventions address the challenges affecting physicians that we identified with high burnout, such as primary care physicians and those practicing at small, rural sites. Policymakers must also recognize and address the high burnout prevalence among physicians practicing in different health systems. The relatively lower burnout rate among VA physicians may guide interventions to address organizational differences between VA and non-VA systems, such as differences in workload and workplace climate. Additional qualitative and mixed-methods research is needed to understand how risk factors for burnout differ based on practice setting.

This study has several limitations. We used two single-item questions from the MBI to identify burnout. While this approach has been validated with other burnout definitions that rely more extensively on the MBI, defining burnout as a dichotomous syndrome may oversimplify the continuum of physician experiences. ${ }^{52}$ Using the existing survey data, we were not able to explore physician perspectives on what caused their burnout or whether they had comorbid depression or suicidal ideation. We used AES responses to identify physicians' service area, but we did not have access to the respondents' specific board certifications. For example, we were not able to discern whether a primary care physician was boarded in family medicine or internal medicine. We had access to limited information on site-level characteristics, and we could not determine details of practice settings, such as staffing and workload for each service areas. In the absence of qualitative and ethnographic data, we were also not able to understand differences in organizational culture between sites. We used a panel data study design to track burnout longitudinally, but we were not able to assess changes in burnout on the individual level because the dataset does not include individual identifiers.

\section{CONCLUSIONS}

Our findings provide key insight into VA physicians affected by burnout, including longitudinal trends, organizational factors, and geographic distribution of burnout. We demonstrated that the prevalence of VA physician burnout has been largely stable in recent years and is lower than reported in many studies of non-VA physicians - though still concerning with burnout consistently affecting more than a third of VA physicians nationally. We also identified site-level characteristics associated with burnout, including higher rates of burnout among rural, non-teaching sites with lower complexity. Additional research is needed to explore the etiology of burnout in different environments and identify effective solutions to address the burnout crisis. Our results can help guide targeted interventions to promote VA physician well-being and may inform future research contrasting the experience of VA and non-VA physicians.

\begin{abstract}
Acknowledgments: We are grateful for the support of VHA National Center for Organization Development for data access to the All Employee Survey.
\end{abstract}

Corresponding Author: Seppo T. Rinne, MD PhD; Center for Healthcare Organization \& Implementation Research, VA, Bedford, MA, USA (e-mail: seppo@bu.edu).

Author Contributions Drs. Rinne and Charns had full access to the data and take responsibility for the integrity of the data and accuracy of the analysis.

Study concept and design: Rinne, Mohr, Wong, Charns

Acquisition, analysis, or interpretation of data: All authors

Drafting of the manuscript: All authors

Critical revision of the manuscript for important data: All authors

Statistical analysis: Mohr

Study supervision: Rinne, Mohr, Charns

Funding Information This study was financially supported in part by an American Thoracic Society Foundation Unrestricted Grant. Dr. Rinne was financially supported by a Parker B. Francis Award and a VA HSR\&D CDA.

\section{Compliance with Ethical Standards:}

The institutional review board at The VA Boston Healthcare System approved the study.

Conflict of Interest: The authors declare that they do not have a conflict of interest.

Role of the Funder/Sponsor: The funder had no role in the design and conduct of the study; collection, management, analysis, or interpretation of the data; preparation, review, or approval of the manuscript; or decision to submit the manuscript for publication.

Disclaimer: The views expressed here are those of the authors and do not necessarily reflect the position or policy of the Department of Veterans Affairs.

\section{REFERENCES}

1. Halbesleben JR, Rathert C. Linking physician burnout and patient outcomes: exploring the dyadic relationship between physicians and patients. Health Care Manage Rev. 2008;33(1):29-39.

2. McHugh MD, Kutney-Lee A, Cimiotti JP, Sloane DM, Aiken LH. Nurses' widespread job dissatisfaction, burnout, and frustration with 
health benefits signal problems for patient care. Health Aff (Millwood). 2011;30(2):202-210

3. Panagioti M, Geraghty $\mathbf{K}$, Johnson $\mathbf{J}$, et al. Association Between Physician Burnout and Patient Safety, Professionalism, and Patient Satisfaction: A Systematic Review and Meta-Analysis. JAMA Intern Med. 2018;178(10): 1317-1331.

4. Maslach C, Leiter MP. Understanding the burnout experience: recent research and its implications for psychiatry. World Psychiatry. 2016;15(2):103-111.

5. Dyrbye LN, Thomas MR, Massie FS, et al. Burnout and suicidal ideation among U.S. medical students. Ann Intern Med. 2008;149(5):334-341.

6. Oreskovich MR, Kaups KL, Balch CM, et al. Prevalence of alcohol use disorders among American surgeons. Arch Surg. 2012;147(2):168-174.

7. Shanafelt TD, Balch CM, Dyrbye L, et al. Special report: suicidal ideation among American surgeons. Arch Surg. 2011;146(1):54-62.

8. Shanafelt TD, Raymond M, Kosty M, et al. Satisfaction with work-life balance and the career and retirement plans of US oncologists. $J$ Clin Oncol. 2014;32(11):1127-1135.

9. Shanafelt T, Sloan J, Satele D, Balch C. Why do surgeons consider leaving practice? J Am Coll Surg. 2011;212(3):421-422.

10. Shanafelt TD, West CP, Sinsky C, et al. Changes in Burnout and Satisfaction With Work-Life Integration in Physicians and the General US Working Population Between 2011 and 2017. Mayo Clin Proc. 2019;94(9):1681-1694

11. Shanafelt TD, Boone S, Tan L, et al. Burnout and satisfaction with worklife balance among US physicians relative to the general US population. Arch Intern Med. 2012;172(18): 1377-1385.

2. Shanafelt TD, Hasan O, Dyrbye LN, et al. Changes in Burnout and Satisfaction With Work-Life Balance in Physicians and the General US Working Population Between 2011 and 2014. Mayo Clin Proc. 2015;90(12): 1600-1613.

13. Rotenstein LS, Torre M, Ramos MA, et al. Prevalence of Burnout Among Physicians: A Systematic Review. JAMA. 2018;320(11):1131-1150.

14. Better Data and Evaluation Could Help Improve Physician Staffing, Recruitment, and Retention Strategies. In: VHA, ed. US Government Accountability Office2017

15. Osatuke K, Draime $\mathbf{J}$, Moore SC, et al. Organization development in the Department of Veterans Affairs. In: Miller T, ed. The Praeger handbook of Veterans Health: History, challenges, issues and developments, Volume IV Future directions in Veterans healthcare. Santa Barbara: Praeger: 2012:21-76.

16. Wooldridge JM. Econometric Analysis of Cross Section and Panel Data, 2nd Edition. Cambridge: The MIT Press; 2018.

17. West CP, Dyrbye LN, Satele DV, Sloan JA, Shanafelt TD. Concurrent validity of single-item measures of emotional exhaustion and depersonalization in burnout assessment. J Gen Intern Med. 2012;27(11):14451452

18. Summary of VHA Facility Complexity Model.

19. VHA and ORH Adopt a New System to Define "Rural". https://www. ruralhealth.va.gov/rural-definition.asp. Accessed January 5, 2019.

20. AAMC Council of Teaching Hospitals and Health Systems. https:// $\mathrm{m}$ e m bers.a a m c.org/e w e b / D yn a mi c P a g e. a s p x ? site=AAMC \&webcode=AAMCOrgSearchResult\&orgtype $=$ Hospital $/$ Health\%20System. Accessed January 5, 2019.

21. Everitt BS, Landau S, Leese M, Stahl D. Cluster Analysis, 5th Edition. King's College London: Wiley 2011.

22. SAS 9.2 [computer program]. SAS Institute Inc.; 2007.

23. R version 3.5.3 (2019-03-11) - "Great Truth" [computer program]

24. 2017 Update: The Complexities of Physician Supply and Demand: Projections From 2015 to 2030: Final Report. In: Colleges AoAM, ed. Washington: IHS, Inc

25. Hussey PS, Ringel JS, Ahluwalia S, et al. Resources and Capabilities of the Department of Veterans Affairs to Provide Timely and Accessible Care to Veterans. Rand Health Q. 2016;5(4):14.

26. VA supports your work-life balance. In. VAntage Point. Vol 2019: Official Blog of the U.S. Department of Veterans Affairs; 2017.

27. Altschuler J, Margolius D, Bodenheimer T, Grumbach K. Estimating a reasonable patient panel size for primary care physicians with teambased task delegation. Ann Fam Med. 2012;10(5):396-400.

28. Tan EC, Chen DR. Second victim: Malpractice disputes and quality of life among primary care physicians. J Formos Med Assoc. 2018.

29. Balch CM, Oreskovich MR, Dyrbye LN, et al. Personal consequences of malpractice lawsuits on American surgeons. J Am Coll Surg. 2011;213(5):657-667
30. Kane L. Medscape National Physician Burnout, Depression \& Suicide Report 2019. https://www.medscape.com/slideshow/2019-lifestyleburnout-depression-6011056. Accessed January 21, 2019.

31. Interim Report: Review of Patient Wait Times, Scheduling Practices, and Alleged Patient Deaths at the Phoenix Health Care System. In: Affairs V, ed. Washington: Department of Veterans Affairs; 2014

32. Osatuke K, Fishman J, Moore SC, Dyrenforth SR. Relationship between Portrayals of VA Hospitals in the Media, and Employee and Patient Satisfaction: An Exploratory Analysis Paper presented at: American Association for Public Opinion Research May 15, 2009, 2009; Hollywood

33. Trivedi RB, Post EP, Sun H, et al. Prevalence, Comorbidity, and Prognosis of Mental Health Among US Veterans. Am $J$ Public Health 2015;105(12):2564-2569.

34. Nelson KM, Helfrich $\mathbf{C}$, Sun $\mathbf{H}$, et al. Implementation of the patientcentered medical home in the Veterans Health Administration: associations with patient satisfaction, quality of care, staff burnout, and hospital and emergency department use. JAMA Intern Med. 2014;174(8):13501358

35. Ladebue AC, Helfrich CD, Gerdes ZT, Fihn SD, Nelson KM, Sayre GG. The experience of Patient Aligned Care Team (PACT) members. Health Care Manage Rev. 2016;41(1):2-10.

36. Meredith LS, Schmidt Hackbarth N, Darling $\mathbf{J}$, et al. Emotional exhaustion in primary care during early implementation of the VA's medical home transformation: Patient-aligned Care Team (PACT). Med Care. 2015;53(3):253-260.

37. Trivedi R, Sylling P, Post EP, et al. Comparing Preventable Hospitalizations Among Veterans With and Without Mental Illnesses Before and After Implementation of PACT. Psychiatr Serv. 2018;69(12):1252-1258. appips201700316.

38. Sylling PW, Wong ES, Liu CF, et al. Patient-centered medical home implementation and primary care provider turnover. Med Care. 2014;52(12):1017-1022.

39. Teixeira-Poit SM, Halpern MT, Kane HL, Keating M, Olmsted M Factors influencing professional life satisfaction among neurologists. BMC Health Serv Res. 2017;17(1):409.

40. Vick B. Analyzing Rural Versus Urban Differences in Career Dissatisfaction and Plans to Leave Among Pennsylvanian Physicians. J Rural Health. 2016;32(2): 164-175

41. Haggerty TS, Fields SA, Selby-Nelson EM, Foley KP, Shrader CD. Physician wellness in rural America: a review. Int $J$ Psychiatry Med. 2013;46(3):303-313.

42. Waddimba AC, Scribani M, Krupa N, May JJ, Jenkins P. Frequency of satisfaction and dissatisfaction with practice among rural-based, groupemployed physicians and non-physician practitioners. BMC Health Serv Res. 2016;16(1):613

43. Tawfik DS, Phibbs CS, Sexton JB, et al. Factors Associated With Provider Burnout in the NICU. Pediatrics. 2017;139(5):e20164134.

44. Mohr DC, Eaton JL, Meterko M, Stolzmann KL, Restuccia JD. Factors associated with internal medicine physician job attitudes in the Veterans Health Administration. BMC Health Serv Res. 2018;18(1):244.

45. Mohr DC, Burgess JF, Jr. Job characteristics and job satisfaction among physicians involved with research in the veterans health administration. Acad Med. 2011;86(8):938-945.

46. Domagala A, Bala MM, Storman D, et al. Factors Associated with Satisfaction of Hospital Physicians: A Systematic Review on European Data. Int $J$ Environ Res Public Health. 2018;15(11):E2546.

47. Weinhold I, Gurtner S. Understanding shortages of sufficient health care in rural areas. Health Policy. 2014;118(2):201-214.

48. Dwyer-Lindgren L, Bertozzi-Villa A. Stubbs RW, et al. Inequalities in Life Expectancy Among US Counties, 1980 to 2014: Temporal Trends and Key Drivers. JAMA Intern Med. 2017;177(7):1003-1011.

49. Spickard A, Jr., Gabbe SG, Christensen JF. Mid-career burnout in generalist and specialist physicians. JAMA. 2002;288(12):1447-1450.

50. Dyrbye LN, Varkey P, Boone SL, Satele DV, Sloan JA, Shanafelt TD. Physician satisfaction and burnout at different career stages. Mayo Clin Proc. 2013;88(12):1358-1367.

51. Panagioti M, Panagopoulou E, Bower $\mathbf{P}$, et al. Controlled Interventions to Reduce Burnout in Physicians: A Systematic Review and Metaanalysis. JAMA Intern Med. 2017;177(2):195-205.

52. The Problem with Cut-Offs for the Maslach Burnout Inventory. Mind Garden; May 31, 2018.

Publisher's Note Springer Nature remains neutral with regard to jurisdictional claims in published maps and institutional affiliations. 